Nils Becker, Christoph Reimann, Dominik Weber, Tobias Lüdtke, Martin Lerch, Thomas Bredow and Richard Dronskowski*

\title{
A density-functional theory approach to the existence and stability of molybdenum and tungsten sesquioxide polymorphs
}

DOI 10.1515/zkri-2016-1960

Received May 25, 2016; accepted August 4, 2016; published online August 26, 2016

\begin{abstract}
The sesquioxides of molybdenum and tungsten have been reported as thin films or on surfaces as early as 1971, but the preparation of bulk materials and their crystal structures are still unknown up to the present day. We present a systematic $a b$ initio approach to their possible syntheses and crystal structures applying complementary methods and basis-set types. For both compounds, the corundum structure is the most stable and does not display any imaginary frequencies. Calculations targeted at a high-pressure synthesis starting from the stable oxides and metals predict a reaction pressure of $15 \mathrm{GPa}$ for $\mathrm{Mo}_{2} \mathrm{O}_{3}$ and over $60 \mathrm{GPa}$ for $\mathrm{W}_{2} \mathrm{O}_{3}$.
\end{abstract}

Keywords: DFT; high-pressure; molybdenum; sesquioxide; tungsten.

\section{Introduction}

The distinct behavior of the $d$-block elements also applies within the chromium group. Accordingly, molybdenum and tungsten are the heavier atoms of this group and show similar atomic and ionic radii which also leads to an alike

\footnotetext{
*Corresponding author: Richard Dronskowski, Institut für Anorganische Chemie, RWTH Aachen University, Landoltweg 1, D-52056 Aachen, Germany; and Jülich-Aachen Research Alliance (JARA-HPC), RWTH Aachen University, D-52074 Aachen, Germany, E-mail: drons@HAL9000.ac.rwth-aachen.de Nils Becker: Institut für Anorganische Chemie, RWTH Aachen University, Landoltweg 1, D-52056 Aachen, Germany; and JülichAachen Research Alliance (JARA-HPC), RWTH Aachen University, D-52074 Aachen, Germany

Christoph Reimann and Thomas Bredow: Mulliken Center for Theoretical Chemistry, Institut für Physikalische und Theoretische Chemie, University of Bonn, Beringstraße 4, D-53115 Bonn, Germany Dominik Weber, Tobias Lüdtke and Martin Lerch: Technische Universität Berlin, Fakultät II, Institut für Chemie, Sekretariat C 2 , Straße des 17. Juni 135, D-10623 Berlin, Germany
}

chemical behavior and is the result (at least in part) of the lanthanoid contraction. Chromium as the lighter atom has a smaller radius and behaves differently, not too surprisingly. The difference between chromium and its heavier homologues molybdenum and tungsten, however, is not as significant as it is in the adjacent vanadium group.

It is quite puzzling to note that the inorganic crystal structure database (ICSD) does not list any sesquioxides of molybdenum or tungsten. Nonetheless, there is a first report on a cubic polymorph of both molybdenum and tungsten sesquioxide by Palatnik et al. [1] which, however, only provides the lattice parameters for the proposed cubic structures, namely $a=6.84 \AA$ for $\mathrm{Mo}_{2} \mathrm{O}_{3}$ and $a=6.86 \AA$ for $\mathrm{W}_{2} \mathrm{O}_{3}$. Second, a composition dubbed $\mathrm{Mo}_{2} \mathrm{O}_{3}$ and $\mathrm{W}_{2} \mathrm{O}_{3}$ was also reportedly obtained on the surface of oxygen-implanted single crystals of molybdenum and tungsten [2]. The observed surface layers, however, were amorphous as a result of oxygen-ion bombardment. Third, the synthesis of tungsten(III) oxide was again claimed as a thin film via atomic layer deposition in 2006 [3] but no details on the crystal structure could be given as the X-ray diffraction data indicated that the samples were amorphous.

As a first experimental attempt, a conventional synthetic approach inspired by the successful synthesis of the metastable sesquioxides of vanadium [4] was used. Different oxide (crystalline and amorphous) and oxide fluoride precursors were reduced with altered $\mathrm{H}_{2} /$ Ar flow rates. The usage of hexafluorides is difficult due to their liquid (Mo) and gaseous (W) aggregate state at ambient conditions. Additionally, experiments with oxide hydrates and oxide nitrides were performed as well. All results were more than underwhelming. Well-known phases such as $\mathrm{MoO}_{2}$ and $\mathrm{WO}_{2}$ were predominantly formed throughout all experiments. Other typical results were highly amorphous samples. Motivated by the lack of experimentally confirmed crystal structures, we used density-functional theory to present a first-principles approach to the crystal structures and possible synthetic routes to molybdenum and tungsten sesquioxide. 


\section{Theoretical methodology}

Periodic density-functional theory (DFT) calculations were performed as implemented in the Vienna $a b$ initio simulation package (VASP) [5]. Core and valence electrons were separated using projector-augmented waves (PAW) [6], and the generalized-gradient-approximation as described by Perdew, Burke and Ernzerhof (GGA-PBE) [7, 8] was used to treat contributions of exchange and correlation. An energy-cutoff of $500 \mathrm{eV}$ and an adapted $k$-point sampling ensured well-converged structures. The $k$-point grid was increased until the number of $k$-points multiplied with the number of atoms was $>5000$, a sufficient criterion in the past; numerical values are given in the SI. All lattice parameters and atomic positions were allowed to relax until the ionic convergence criterion of $10^{-6} \mathrm{eV}$ was reached. For selected cases (i.e. $\mathrm{Al}_{2} \mathrm{O}_{3}$ as the reference structure, $\mathrm{La}_{2} \mathrm{O}_{3}$ and $\mathrm{Sb}_{2} \mathrm{O}_{3}$ ) additional corrections for van-der-Waals forces were included, namely Grimme's "D3(BJ)" dispersion correction with Becke-Johnson damping $[9,10]$ as implemented in VASP 5.3.5.

To investigate the unknown crystal structures of the hypothetical compounds $\mathrm{Mo}_{2} \mathrm{O}_{3}$ and $\mathrm{W}_{2} \mathrm{O}_{3}$, a set of 13 well-known $\mathrm{A}_{2} \mathrm{X}_{3}$ structures was chosen as starting structures, namely the same used in our previous studies on the sesquioxides of $\mathrm{Cr}, \mathrm{Nb}$ and Ta. A full relaxation of the structures including atomic positions and cell parameters was performed to ensure the lowest-energy structure to be found. To speed up the calculations, symmetry was applied while iterating but no explicit symmetry reduction or change to subgroups was introduced, unless automatically occuring during optimization. To predict high-pressure polymorphs, the volume of the optimized structures at ambient pressure was varied by scaling the cell parameters from $94 \%$ to $104 \%$, and the resulting energy vs. volume data were fitted to the Birch-Murnaghan equation of state [11]. Thus, the pressure and, eventually, the reaction enthalpies as a function of the pressure were calculated.

The dynamic stability of selected $\mathrm{M}_{2} \mathrm{O}_{3}$ candidates was investigated by calculating the phonon density of states (pDOS) using the program Phonopy [12]. As a first step, supercells of the optimized structures were constructed. All symmetry-inequivalent atoms were then slightly shifted out of their equilibrium positions to numerically calculate the Hellmann-Feynman forces. Subsequently, the force constants and then the dynamical matrices were computed. Phonon frequencies and eigenvectors were calculated from the dynamical matrices. The pDOS can then be assessed by summing up over all phonon frequencies [13].
As a complementary approach and in order to investigate the influence of exact exchange on the electronic ground states and the relative stabilities of the polymorphs, we also calculated the same set of 13 structures with the crystalline-orbital program CRYSTAL14 [14]. Here, the Bloch functions are represented by atom-centered basis functions. The localized nature of the basis functions leads to an efficient calculation of exact exchange. For comparison with the VASP-PBE results, we employed the hybrid functional PW1PW which has been previously used in similar studies of thermodynamic and electronic properties of transition-metal oxides $[15,16]$. The HartreeFock contribution of this functional is $20 \%$, and it was optimized towards the band gaps of $\mathrm{MgO}, \mathrm{NiO}$, and $\mathrm{CoO}$ [17]. The basis sets remained unmodified. Atomic basis sets of standard quality were selected for Mo [18], W [19] and $O$ [20]. We then considered closed-shell diamagnetic (DM), open-shell ferromagnetic (FM) and antiferromagnetic (AFM) states. Full optimizations within the symmetry restrictions of the space groups and their magnetic subgroups were performed. In what follows in tabular form, only the relative energies of the lowest magnetic states of the polymorphs are given. Magnetic calculations with FM and AFM ordering resulted in zero magnetic moments and identical energy values for the stable corundum type structure when using VASP.

\section{Results and discussion}

As said before, a set of 13 well-known $\mathrm{A}_{2} \mathrm{X}_{3}$ starting structures was first fully optimized for both compositions $\mathrm{Mo}_{2} \mathrm{O}_{3}$ and $\mathrm{W}_{2} \mathrm{O}_{3}$ with VASP-PBE and CRYSTAL-PW1PW. The resulting energies and volumes are listed in Table 1 for the molybdenum and Table 2 for the tungsten case. The corundum-type $\left[\mathrm{Al}_{2} \mathrm{O}_{3}\right]$ starting structure resulted in the lowest total energy for both elements. The fully relaxed structure resembles the corundum starting structure quite well but with one significant difference: the previously slightly distorted hexagonal close packing (hcp) of the oxygen atoms becomes perfectly ordered in the relaxed structure. The origin of this effect could not be determined yet, but a magnetic effect is unlikely, as a magnetic moment was unstable in the calculations. When performing the identical relaxation with $\mathrm{Al}_{2} \mathrm{O}_{3}$ in the corundum structure, the ordering phenomenon was not observed. Therefore, a temperature effect also seems unlikely. Furthermore, the monoclinic $\left[\mathrm{V}_{2} \mathrm{O}_{3}\right]$ starting structure (Figure 1, right) transforms into the corundum structure upon structural optimization and, thus, arrives 
Tab. 1: Molar volumes and relative energies per formula unit for hypothetical molybdenum sesquioxide $\mathrm{Mo}_{2} \mathrm{O}_{3}$ polymorphs as a function of computational methodology and magnetic state (DM, diamagnetic; FM, ferromagnetic; AFM, antiferromagnetic).

\begin{tabular}{|c|c|c|c|c|c|}
\hline Structure type & Reference & $V(\mathrm{PBE})\left(\AA^{3}\right)$ & $\Delta E(\mathrm{PBE})(\mathrm{eV})$ & $\Delta E(\mathrm{PBE}-\mathrm{D} 3)(\mathrm{eV})$ & $\Delta E(\mathrm{PW} 1 \mathrm{PW})(\mathrm{eV})$ \\
\hline $\mathrm{Al}_{2} \mathrm{O}_{3}$ & [21] & 54.02 & 0.00 & 0.00 & 0.00 (AFM) \\
\hline $\mathrm{V}_{2} \mathrm{O}_{3}$ & {$[22]$} & 54.02 & 0.00 & & $0.00(\mathrm{DM})$ \\
\hline $\mathrm{Ga}_{2} \mathrm{O}_{3}$ & [23] & 55.22 & 0.30 & & $0.61(\mathrm{DM})$ \\
\hline $\mathrm{Rh}_{2} \mathrm{O}_{3}$ & [24] & 53.51 & 0.51 & & 0.51 (AFM) \\
\hline $\mathrm{Sm}_{2} \mathrm{O}_{3}$ & {$[25]$} & 57.15 & 0.69 & & $1.21(\mathrm{FM})$ \\
\hline $\mathrm{Sb}_{2} \mathrm{O}_{3}$ & [26] & 61.75 & 0.70 & 0.90 & 0.80 (AFM) \\
\hline $\mathrm{Au}_{2} \mathrm{O}_{3}$ & [27] & 53.54 & 0.95 & & 2.12 (AFM) \\
\hline $\mathrm{Fe}_{2} \mathrm{O}_{3}$ & {$[28,29]$} & 57.56 & 1.04 & & 1.93 (AFM) \\
\hline $\mathrm{Sb}_{2}^{2} \mathrm{~S}_{3}$ & [30] & 58.08 & 1.39 & & 1.01 (AFM) \\
\hline $\mathrm{GdFeO}_{3}$ & [31] & 53.07 & 1.94 & & 1.45 (AFM) \\
\hline $\mathrm{La}_{2} \mathrm{O}_{3}$ & [32] & 60.99 & 1.98 & 2.11 & 1.59 (AFM) \\
\hline Bixbyite & [33] & 56.47 & 2.07 & & 1.34 (AFM) \\
\hline $\mathrm{Ag}_{2} \mathrm{O}_{3}$ & [34] & 57.65 & 2.84 & & 1.77 (AFM) \\
\hline
\end{tabular}

The listed structures represent the starting structures.

Tab. 2: Same as before but for $\mathrm{W}_{2} \mathrm{O}_{3}$.

\begin{tabular}{|c|c|c|c|c|c|}
\hline Structure type & Reference & $V(\mathrm{PBE})\left(\AA^{3}\right)$ & $\Delta E(\mathrm{PBE})(\mathrm{eV})$ & $\Delta E(\mathrm{PBE}-\mathrm{D} 3)(\mathrm{eV})$ & $\Delta E(P W 1 P W)(e V)$ \\
\hline $\mathrm{Al}_{2} \mathrm{O}_{3}$ & {$[21]$} & 54.50 & 0.00 & 0.00 & 0.00 (AFM) \\
\hline $\mathrm{V}_{2} \mathrm{O}_{3}$ & [22] & 54.50 & 0.00 & & 0.00 (AFM) \\
\hline $\mathrm{Rh}_{2} \mathrm{O}_{3}$ & [24] & 54.01 & 0.50 & & 1.04 (AFM) \\
\hline $\mathrm{Sm}_{2} \mathrm{O}_{3}$ & [25] & 58.00 & 0.66 & & 2.04 (AFM) \\
\hline $\mathrm{Ga}_{2} \mathrm{O}_{3}$ & [23] & 58.79 & 0.91 & & 1.38 (AFM) \\
\hline $\mathrm{Fe}_{2} \mathrm{O}_{3}^{3}$ & {$[28,29]$} & 59.56 & 0.98 & & 1.12 (AFM) \\
\hline $\mathrm{Au}_{2}^{2} \mathrm{O}_{3}$ & [27] & 53.98 & 1.23 & & 0.49 (AFM) \\
\hline $\mathrm{Sb}_{2}^{2} \mathrm{~S}_{3}$ & [30] & 59.47 & 1.66 & & 0.42 (AFM) \\
\hline $\mathrm{Sb}_{2} \mathrm{O}_{3}$ & [26] & 57.37 & 1.77 & 0.14 & 0.34 (AFM) \\
\hline $\mathrm{GdFeO}_{3}$ & [31] & 52.73 & 2.20 & & 0.82 (AFM) \\
\hline $\mathrm{La}_{2} \mathrm{O}_{3}$ & [32] & 62.03 & 2.28 & 2.42 & 1.18 (AFM) \\
\hline Bixbyite & [33] & 56.75 & 2.49 & & 0.76 (AFM) \\
\hline $\mathrm{Ag}_{2} \mathrm{O}_{3}$ & [34] & 57.62 & 2.89 & & 1.04 (AFM) \\
\hline
\end{tabular}

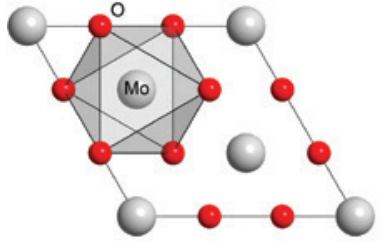

Corundum-type

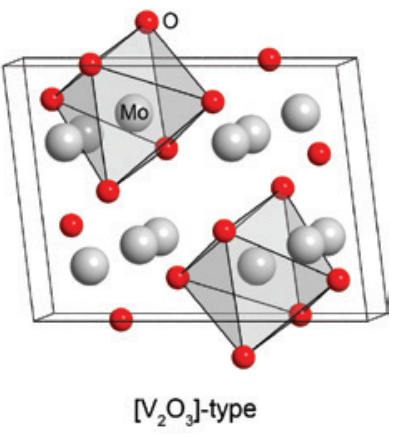

Fig. 1: Relaxed crystal structure of $\mathrm{Mo}_{2} \mathrm{O}_{3}\left(\right.$ and $\mathrm{W}_{2} \mathrm{O}_{3}$ ) in the corundum structure (left) displaying the lowest energy. The $\left[\mathrm{V}_{2} \mathrm{O}_{3}\right]$ starting structure (right) transforms into the corundum structure (left) upon structural relaxation.

at the same total energy. Once again, this applies to both sesquioxides of molybdenum and tungsten which is why we only depict the structure of $\mathrm{Mo}_{2} \mathrm{O}_{3}$ in Figure 1. We also note that this behavior $\left(\mathrm{V}_{2} \mathrm{O}_{3} \rightarrow \mathrm{Al}_{2} \mathrm{O}_{3}\right)$ has previously been observed in a similar DFT investigation for chromium sesquioxide [35], in which the $\left[\mathrm{V}_{2} \mathrm{O}_{3}\right.$ ] starting structure also transformed into the corundum structure upon structural optimization. No starting structure type seems to be within a reasonable energetic range of about $0.2 \mathrm{eV}$ $(\approx 20 \mathrm{~kJ} / \mathrm{mol}$ per formula unit) to the corundum structure type which is the energetically preferred structure for both investigated sesquioxides. This is also true when the CRYSTAL-PW1PW results are considered. Despite the fact that the magnetic ground states derived from VASPPBE and CRYSTAL-PW1PW are different in many cases, which then leads to a different ordering of the less stable polymorphs, the corundum and $\left[\mathrm{V}_{2} \mathrm{O}_{3}\right]$-derived structures are clearly preferred over all the others. The stability differences between these structures and other phases increases even more whenever London dispersion interactions (needed in particular for layered structures) are 
taken into account. Therefore, and for reasons of computational efficiency, we will continue our investigation only with the corundum structure type. The calculated cell parameters and atomic positions for $\mathrm{Mo}_{2} \mathrm{O}_{3}$ and $\mathrm{W}_{2} \mathrm{O}_{3}$ are listed in Table 3.

For the most stable corundum polymorphs of both $\mathrm{Mo}_{2} \mathrm{O}_{3}$ and $\mathrm{W}_{2} \mathrm{O}_{3}$ the electronic density of states was calculated with CRYSTAL-PW1PW. This is because, quite generally, hybrid methods provide a more accurate description of the electronic structure compared to semilocal GGA functionals. In Figure 2, the projected density of states of the highest valence and lowest conduction bands are shown for the AFM ground states.

The electronic structures of the two compounds are rather similar. In both cases, the valence band and the conduction band predominantly consist of metal $4 d(5 d)$ orbitals, respectively, but with significant contributions from

Tab. 3: Structural details and molar volumes of molybdenum and tungsten sesquioxide in the corundum structure based on VASP-PBE calculations.

\begin{tabular}{cllrrr}
\hline & $R 3 C(167)$ & Atom & $\boldsymbol{x}$ & $\boldsymbol{y}$ & $\boldsymbol{z}$ \\
\hline $\mathrm{Mo}_{2} \mathrm{O}_{3}$ & & & & & \\
$a$ & $4.710 \AA$ & Mo & 0 & 0 & 0.163 \\
$c$ & $16.874 \AA$ & 0 & $1 / 3$ & 0 & $1 / 4$ \\
$Z$ & 6 & & & & \\
$V$ & $54.01 \AA^{3}$ & & & & \\
$\mathrm{~W}_{2} \mathrm{O}_{3}$ & & & & & \\
$a$ & $4.663 \AA$ & $\mathrm{W}$ & 0 & 0 & 0.163 \\
$c$ & $17.366 \AA$ & 0 & $1 / 3$ & 0 & $1 / 4$ \\
$Z$ & 6 & & & & \\
$V$ & $54.50 \AA^{3}$ & & & & \\
\hline
\end{tabular}

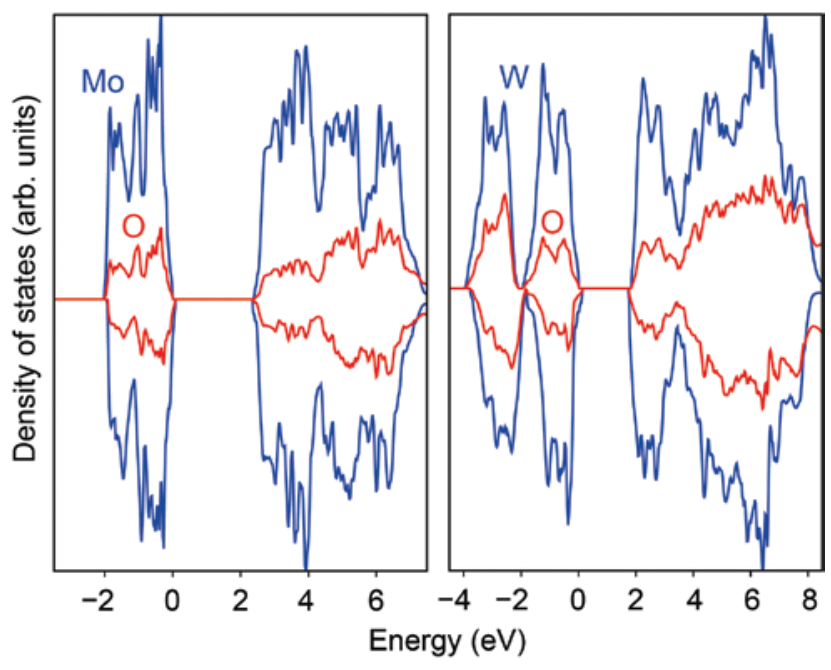

Fig. 2: Projected density of states for both sesquioxides in the corundum structure according to CRYSTAL-PW1PW. The orbital energy is given relative to the Fermi energy at zero eV. the $\mathrm{O} 2 p$ states, indicating a partially covalent bonding. The upper part of the valence band of $\mathrm{W}_{2} \mathrm{O}_{3}$ (right frame) is split into two domains from 0 to $-2 \mathrm{eV}$ and between -2 and $-4 \mathrm{eV}$ below the Fermi energy. $\mathrm{Mo}_{2} \mathrm{O}_{3}$ (left frame) only shows one domain between 0 and $-2 \mathrm{eV}$. This is possibly due to a smaller distortion from octahedral site symmetry of the metal ions in $\mathrm{Mo}_{2} \mathrm{O}_{3}$ so that the splitting of the $t_{2 g}$ orbitals is smaller in this compound. The fundamental band gap of $\mathrm{Mo}_{2} \mathrm{O}_{3}(2.39 \mathrm{eV})$ is slightly larger than that of $\mathrm{W}_{2} \mathrm{O}_{3}(1.84 \mathrm{eV})$ at PW1PW level of theory. If the calculated electronic band gaps are a reasonable approximation to the optical band gaps, i.e. if the excitonic effects are small, then molybdenum sesquioxide could be a promising candidate for photocatalytical applications since the gap is in the optimal region, e.g. for water splitting.

Prior to an investigation of possible synthetic routes, we performed phonon calculations on the sesquioxides of molybdenum and tungsten with VASP-PBE taking the corundum structure. The calculated phonon densities of states (pDOS) depicted in Figure 3 show no imaginary modes for both sesquioxides and thereby verify their dynamic stability.

Having identified the corundum structure as the most likely one for $\mathrm{Mo}_{2} \mathrm{O}_{3}$ and $\mathrm{W}_{2} \mathrm{O}_{3}$, we then considered various reaction routes to estimate the feasibility of their syntheses. The selected pathways are limited to solid-state materials and only include the metals and their different oxides. Molybdenum and tungsten both only form two oxides with an integer oxidation state, namely the dioxide and the trioxide, and therefore allowing only two simple reaction routes to be computationally explored:
(I) $\mathrm{M}+\mathrm{MO}_{3} \rightarrow \mathrm{M}_{2} \mathrm{O}_{3}$
(II) $1 / 2 \mathrm{M}+3 / 2 \mathrm{MO}_{2} \rightarrow \mathrm{M}_{2} \mathrm{O}_{3}$

The corresponding reaction enthalpies and volumes relative to the reactants are listed in Table 4.

When investigating such reaction enthalpies, one has to be aware of two key issues: first, the reactions are always equilibrium reactions and, second, they compete with each other. For example: if reaction (I) is exothermic, the reactants will yield the product of route (I). This product, however, will decay into the reactants of reaction (II) in case the latter is endothermic. As a consequence, all possible reaction routes must show exothermic reaction enthalpies for the proposed synthetic conditions.

The synthesis of $\mathrm{Mo}_{2} \mathrm{O}_{3}$ via the trioxide route (I) is exothermic while route II shows a positive reaction enthalpy of ca. $37 \mathrm{~kJ} / \mathrm{mol}$. Also, the large volume loss of the product relative to the reactants calls for a high-pressure synthesis. For $\mathrm{W}_{2} \mathrm{O}_{3}$, both reaction routes show very similar reaction 

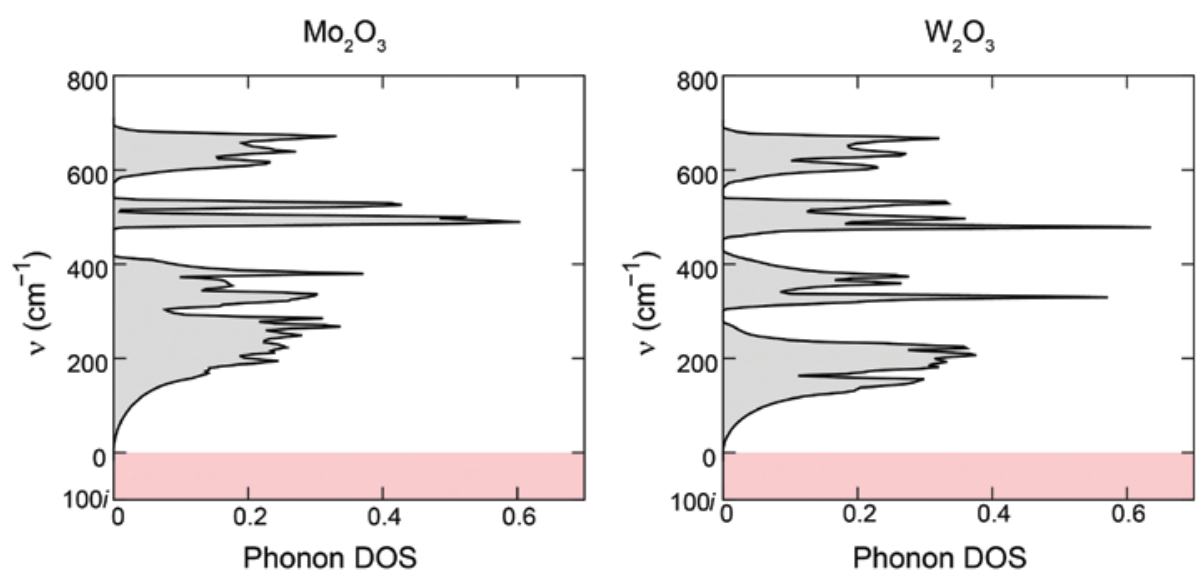

Fig. 3: Calculated phonon DOS of both sesquioxides in the corundum structure based on VASP-PBE results.

Tab. 4: Relative theoretical reaction enthalpies and volumes of $\mathrm{M}_{2} \mathrm{O}_{3}$ for the hypothetical reaction routes I and II based on VASP-PBE results.

\begin{tabular}{|c|c|c|c|c|}
\hline \multirow[t]{2}{*}{ Synthesis } & \multicolumn{2}{|r|}{$\mathrm{Mo}_{2} \mathrm{O}_{3}$} & \multicolumn{2}{|r|}{$\mathrm{W}_{2} \mathrm{O}_{3}$} \\
\hline & $\Delta V\left(\AA^{3}\right)$ & $\Delta H_{\mathrm{R}}(\mathrm{kJ} / \mathrm{mol})$ & $\Delta V\left(\AA^{3}\right)$ & $\Delta H_{\mathrm{R}}(\mathrm{kJ} / \mathrm{mol})$ \\
\hline (I) $\mathrm{M}+\mathrm{MO}_{3} \rightarrow \mathrm{M}_{2} \mathrm{O}_{3}$ & -20.30 & -89.26 & -19.38 & 105.91 \\
\hline (II) $1 / 2 \mathrm{M}+3 / 2 \mathrm{MO}_{2} \rightarrow \mathrm{M}_{2} \mathrm{O}_{3}$ & -4.56 & 37.06 & -4.16 & 104.62 \\
\hline
\end{tabular}

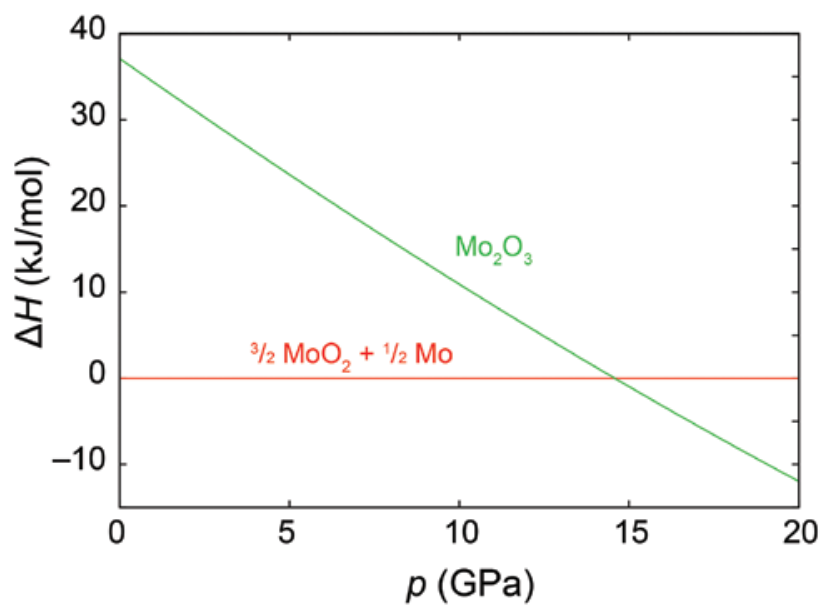

Fig. 4: Density-functional (VASP-PBE) enthalpy-pressure diagram for the synthesis of $\mathrm{Mo}_{2} \mathrm{O}_{3}$ in the corundum structure via reaction II.

enthalpies of about $105 \mathrm{~kJ} / \mathrm{mol}$ and a negative relative volume. Hence, only a high-pressure synthesis is a good choice for the tungsten sesquioxides, as well.

To calculate the pressure dependence of all materials, the energy vs. volume curves were calculated for all reactants and products. By fitting the results to the BirchMurnaghan equation of state [11] and subsequent differentiation, the energy dependence of the pressure is obtained.

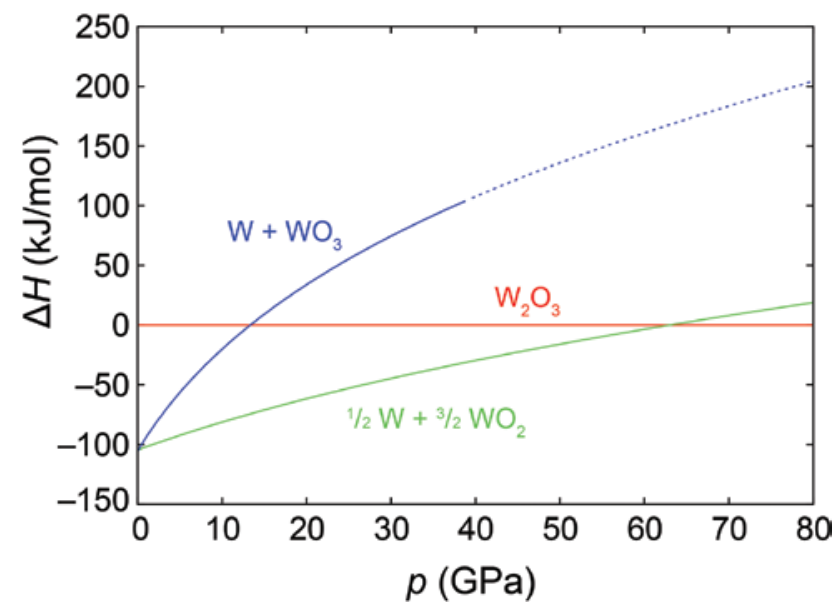

Fig. 5: Density-functional (VASP-PBE) enthalpy-pressure diagram for the synthesis of $\mathrm{W}_{2} \mathrm{O}_{3}$ via reaction I (blue, extrapolated for $p>40$ $\mathrm{GPa}$ ) and reaction II (green).

The pressure-dependent enthalpies can be obtained via $H=E+p V$ which are depicted in Figures 4 and 5 relative to the corresponding reactants. According to Figure 4, $\mathrm{Mo}_{2} \mathrm{O}_{3}$ should form around $15 \mathrm{GPa}$, not necessarily a routine high-pressure experiment, but still manageable. As mentioned in the introduction, it is impossible to synthesize $\mathrm{Mo}_{2} \mathrm{O}_{3}$ via a conventional synthesis route at ambient pressure. Reduction of $\mathrm{MoO}_{3}, \mathrm{MoO}_{3} \cdot \mathrm{H}_{2} \mathrm{O}$, molybdenum oxide 
nitrides, and molybdenum oxide fluorides with various $\mathrm{H}_{2} / \mathrm{Ar}$ gas mixtures at temperatures above $548 \mathrm{~K}$ always resulted in the formation of $\mathrm{MoO}_{2}$ and more complex phases like $\mathrm{Mo}_{4} \mathrm{O}_{11}$ or $\mathrm{Mo}_{18} \mathrm{O}_{52}$.

As shown in Figure 5, the two possible reaction routes for $\mathrm{W}_{2} \mathrm{O}_{3}$ require quite different pressures. At a pressure of about $12 \mathrm{GPa}$ the sesquioxide becomes more favorable than the reactants of route I, while route II needs a pressure of more than $60 \mathrm{GPa}$ to yield the sesquioxide. Of course, this huge difference is a direct consequence of the more negative relative volume of reaction I (Table 4). An attempt to prepare bulk $\mathrm{W}_{2} \mathrm{O}_{3}$ therefore requires very high pressures of over $60 \mathrm{GPa}$ and is experimentally quite challenging. The role of the entropy is rather small for both sesquioxides: at $300 \mathrm{~K}$ the entropy term is only about $2 \mathrm{~kJ} /$ mol for the relevant dioxide route (II) and, thus, the influence on the transition pressures is tiny (under $1 \mathrm{GPa}$ ). All these findings are reflected by our unsuccessful attempts in synthesizing this phase at ambient pressure. The range of used precursors and reaction conditions were comparable to those of the lighter homologue. Additionally, different oxide chlorides were also tested. Summarizing the results, it can be stated that even at the lowest possible $\mathrm{H}_{2}$ flow rate $(0.01 \mathrm{~L} / \mathrm{h})$, reduction of the precursor to tungsten metal was observed at temperatures above $873 \mathrm{~K}$. At lower temperatures, $\mathrm{WO}_{2}$ was the main product.

\section{Conclusion}

Density-functional theory calculations by means of the GGA and a hybrid level on different sesquioxide structures with the composition $\mathrm{Mo}_{2} \mathrm{O}_{3}$ and $\mathrm{W}_{2} \mathrm{O}_{3}$ yield the corundum type as the structure with the lowest energy. Upon structural optimization, the monoclinic $\left[\mathrm{V}_{2} \mathrm{O}_{3}\right]$ structure transforms into the corundum structure, as was previously also observed for $\mathrm{Cr}_{2} \mathrm{O}_{3}$. All other investigated structures are energetically much less favorable and therefore are not likely to be found for the sesquioxides of molybdenum and tungsten. Hypothetical synthetic routes were analyzed to estimate the feasibility of the preparation. The synthesis of $\mathrm{Mo}_{2} \mathrm{O}_{3}$ should be possible via the dioxide at a pressure above $15 \mathrm{GPa}$, while a synthesis of $\mathrm{W}_{2} \mathrm{O}_{3}$ requires a transition pressure $>60 \mathrm{GPa}$ and should be accomplished via the dioxide, as well. Such high-pressure experiments are in progress. Phonon calculations concerning the dynamic stability of both sesquioxides suggest that they are stable.

Acknowledgments: This work was supported by the DFG within the priority program SPP 1415. We thank the computing center at RWTH Aachen University for providing large amounts of CPU time.

\section{References}

[1] L. S. Palatnik, O. A. Obol'yaninova, M. N. Naboka, N. T. Gladkikh, New tungsten oxide modifications. Izvestiya Akademii Nauk SSSR, Neorganicheskie Materialy 1973, 9, 801.

[2] B. G. Eristavi, E. M. Diasamidze, R. N. Dekanosidze, N. I. Maisuradze, E. R. Kutelia, A. V. Sichinava, N. E. Menabde, Structural transformations of oxygen implanted surfaces of refractory metals (Mo, W, Nb). Acta Metall. Mater. 1991, 39, 1703.

[3] C. L. Dezelah, O. M. El-Kadri, I. M. Szilágyi, J. M. Campbell, K. Arstila, L. Niinistö, C. H. Winter, Atomic layer deposition of tungsten(III) oxide thin films from $\mathrm{W}_{2}\left(\mathrm{NMe}_{2}\right)_{6}$ and water: precursor-based control of oxidation state in the thin film material. J. Am. Chem. Soc. 2006, 128, 9638.

[4] D. Weber, A. Stork, S. Nakhal, C. Wessel, C. Reimann, W. Hermes, A. Müller, T. Ressler, R. Pöttgen, T. Bredow, R. Dronskowski, M. Lerch, Bixbyite-Type $\mathrm{V}_{2} \mathrm{O}_{3}$ - a metastable polymorph of vanadium sesquioxide. Inorg. Chem. 2011, 50, 6762.

[5] G. Kresse, J. Furthmüller, Efficiency of ab-initio total energy calculations for metals and semiconductors using a plane-wave basis set. Comput. Mater. Sci. 1996, 6, 15.

[6] P. E. Blöchl, Projector augmented-wave method. Phys. Rev. B 1994, 50, 17953.

[7] J. P. Perdew, K. Burke, M. Ernzerhof, Generalized gradient approximation made simple [Phys. Rev. Lett. 77, 3865 (1996)]. Phys. Rev. Lett. 1997, 78, 1396.

[8] J. P. Perdew, K. Burke, M. Ernzerhof, Generalized gradient approximation made simple. Phys. Rev. Lett. 1996, 77, 3865.

[9] S. Grimme, J. Antony, S. Ehrlich, H. Krieg, A consistent and accurate ab initio parametrization of density functional dispersion correction (DFT-D) for the 94 elements H-Pu. J. Chem. Phys. 2010, 132, 154104.

[10] S. Grimme, S. Ehrlich, L. Goerigk, Effect of the damping function in dispersion corrected density functional theory. J Comput. Chem. 2011, 32, 1456.

[11] F. Birch, Finite elastic strain of cubic crystals. Phys. Rev. 1947, $71,809$.

[12] A. Togo, F. Oba, I. Tanaka, First-principles calculations of the ferroelastic transition between rutile-type and $\mathrm{CaCl}_{2}$-type $\mathrm{SiO}_{2}$ at high pressures. Phys. Rev. B 2008, 78, 134106.

[13] R. P. Stoffel, C. Wessel, M.-W. Lumey, R. Dronskowski, Ab initio thermochemistry of solid-state materials. Angew. Chem. Int. Ed. 2010, 49, 5242.

[14] R. Dovesi, R. Orlando, A. Erba, C. M. Zicovich-Wilson, B. Civalleri, S. Casassa, L. Maschio, M. Ferrabone, M. De La Pierre, P. D’Arco, Y. Noël, M. Causà, M. Rérat, B. Kirtman, CRYSTAL14: a program for the ab initio investigation of crystalline solids. Int. J. Quantum Chem. 2014, 114, 1287.

[15] C. Wessel, C. Reimann, A. Müller, D. Weber, M. Lerch, T. Ressler, T. Bredow, R. Dronskowski, Electronic structure and thermodynamics of $\mathrm{V}_{2} \mathrm{O}_{3}$ polymorphs. J. Comput. Chem. 2012, 33, 2102.

[16] H. Wolff, T. Bredow, M. Lerch, H. Schilling, E. Irran, A. Stork, R. Dronskowski, A first-principles study of the electronic and 
structural properties of $\gamma$-TaON. J. Phys. Chem. A 2007, 111, 2745.

[17] T. Bredow, A. R. Gerson, Effect of exchange and correlation on bulk properties of MgO, NiO, and CoO. Phys. Rev. B 2000, 61, 5194.

[18] F. Cora, A. Patel, N. M. Harrison, C. Roetti, C. Richard A. Catlow, An ab initio Hartree-Fock study of $\alpha-\mathrm{MoO}_{3}$. J. Mater. Chem. 1997, 7, 959.

[19] F. Corà, A. Patel, N. M. Harrison, R. Dovesi, C. R. A. Catlow, An ab initio Hartree-Fock study of the cubic and tetragonal phases of bulk tungsten trioxide. J. Am. Chem. Soc. 1996, 118, 12174.

[20] A. M. Ferrari, C. Pisani, An ab initio periodic study of NiO supported at the $\operatorname{Pd}(100)$ surface. Part 1: the perfect epitaxial monolayer. J. Phys. Chem. B 2006, 110, 7909.

[21] E. Newnham, Y. M de. Haan, Refinement of the $\mathrm{Al}_{2} \mathrm{O}_{3}, \mathrm{Ti}_{2} \mathrm{O}_{3}, \mathrm{~V}_{2} \mathrm{O}_{3}$ and $\mathrm{Cr}_{2} \mathrm{O}_{3}$ structures. Z. Kristallogr. 1962, 117, 235.

[22] P. D. Dernier, M. Marezio, Crystal structure of the low-temperature antiferromagnetic phase of $\mathrm{V}_{2} \mathrm{O}_{3}$. Phys. Rev. B 1970, 2, 3771.

[23] J. Ahman, G. Svensson, J. Albertsson, A reinvestigation of B-gallium oxide. Acta Crystallogr., Sect. C 1996, 52, 1336.

[24] R. D. Shannon, C. T. Prewitt, Synthesis and structure of a new high-pressure form of $\mathrm{Rh}_{2} \mathrm{O}_{3}$. J. Solid State Chem. 1970, 2, 134.

[25] D. T. Cromer, The crystal structure of monoclinic $\mathrm{Sm}_{2} \mathrm{O}_{2}$. J. Phys. Chem. 1957, 61, 753.

[26] C. Svensson, The crystal structure of orthorhombic antimony trioxide, $\mathrm{Sb}_{2} \mathrm{O}_{3}$. Acta Crystallogr., Sect. B 1974, 30, 458.
[27] P. G. Jones, H. Rumpel, E. Schwarzmann, G. M. Sheldrick, H. Paulus, Gold(III) oxide. Acta Crystallogr., Sect. B 1979, 35, 1435.

[28] E. Tronc, C. Chanéac, J. P. Jolivet, Structural and magnetic characterization of $\varepsilon-\mathrm{Fe}_{2} \mathrm{O}_{3}$. J. Solid State Chem. 1998, 139, 93.

[29] L. Smrčok, V. Langer, M. Halvarsson, S. Ruppi, A new Rietveld refinement of $\mathrm{K}-\mathrm{Al}_{2} \mathrm{O}_{3}$. Z. Kristallogr. 2001, 216, 409.

[30] A. Kyono, M. Kimata, Structural variations induced by difference of the inert pair effect in the stibnite-bismuthinite solid solution series (Sb,Bi) ${ }_{2} \mathrm{~S}_{3}$. Am. Mineral. 2004, 89, 932.

[31] S. Geller, Crystal structure of gadolinium orthoferrite, $\mathrm{GdFeO}_{3}$. J. Chem. Phys. 1956, 24, 1236.

[32] W. C. Koehler, E. O. Wollan, Neutron-diffraction study of the structure of the A-form of the rare earth sesquioxides. Acta Crystallogr. 1953, 6, 741.

[33] S. Nakhal, W. Hermes, T. Ressler, R. Pöttgen, M. Lerch, Synthesis, crystal structure and magnetic properties of bixbyite-type vanadium oxide nitrides. $Z$. Naturforsch. $B$ 2009, 64, 281.

[34] B. Stehlík, P. Weidenthaler, J. Vlach: Kristallstruktur von Silber(III)-Oxyd. Collect. Czech. Chem. Commun. 1959, 24, 1581.

[35] C. Wessel, R. Dronskowski, A first-principles study on chromium sesquioxide, $\mathrm{Cr}_{2} \mathrm{O}_{3}$. J. Solid State Chem. 2013, 199, 149.

Supplemental Material: The online version of this article (DOI: 10.1515/zkri-2016-1960) offers supplementary material, available to authorized users. 